\title{
Spectroscopic Analysis of HR 1099
}

\author{
I.S. Savanov ${ }^{1}$, I. Tuominen ${ }^{2}$ \\ ${ }^{1}$ Crimean Astrophysical Observatory, Nauchny, 334413, Crimea, USSR \\ ${ }^{2}$ Observatory and Astrophysics Laboratory, University of Helsinki, \\ Tähtitorninmäki, 00130 Helsinki, Finland
}

\begin{abstract}
We have calculated synthetic spectra for HR 1099 for the wavelength region $\lambda 6425-6445 \AA$, which includes the CaI $\lambda 6439 \AA$ and Fel $\lambda 6430 \AA$ lines, often used for the surface imaging of rapidly rotating late-type stars. The best agreement between the theoretical and observed spectra can be achieved with solar abundances in the both components of the system, except for $\mathrm{Ca}$ in the primary. Theoretical calculations give the possibility of estimating the influence of microturbulence, damping and blending on $\mathrm{Ca} \mathrm{I}$ and $\mathrm{Fe} I$ line profiles.
\end{abstract}

HR 1099 (V711 Tau) is one of the most investigated RS CVn type stars. Our spectroscopic analysis is based on the spectra of HR 1099 that can be found in the literature (Fekel, 1983; Vogt, 1983). Observations were analysed using model atmospheres from a grid of Bell et al. (1975) with additional models obtained from $\mathrm{K}$. Eriksson for the temperature range $5500-4000 \mathrm{~K}$ and gravities 3.00-4.50. Following the results by Fekel (1983), we used models with the following parameters: $T_{\text {eff }}=4500 \mathrm{~K}, \log g=3.75$ for the primary and $T_{\text {eff }}=5500 \mathrm{~K}, \log g=4.5$ for the secondary. The values of $v \sin i$ were adopted from Vogt and Penrod (1982): 38 $\mathrm{km} / \mathrm{s}$ for the primary and $13 \mathrm{~km} / \mathrm{s}$ for the secondary.

A composite spectrum of the double system at arbitrary phase was synthesized by the methods described by Lyubimkov and Samedov (1987) - see Fig. 1. The radii of the components and the radial velocity curve were published by Fekel (1983). From the results of our calculations for the spectral region $\lambda 6425-6445 \AA$ (Fig. 2) we can conclude that elements such as $\mathrm{Fe}$ and $\mathrm{Ca}$, the lines of which are best represented in this region, have abundances close to solar in the secondary component. The best fit with the profiles of the chromospherically active component can be obtained with solar abundances of $\mathrm{Fe}$, but the abundance of $\mathrm{Ca}$ must be reduced by 0.5 dex. The remaining discrepancy between the observed and theoretical profiles may be connected with the inaccuracy of atmospheric parameters, atomic data for the spectral lines and with the existence of spotted areas on the surface of the star. In the present report we use the standard method for the rotational broadening of the lines (e.g. Gray, 1976, p. 394), without a calculation of the local line profile explicitely on the visible disc for different distances from 

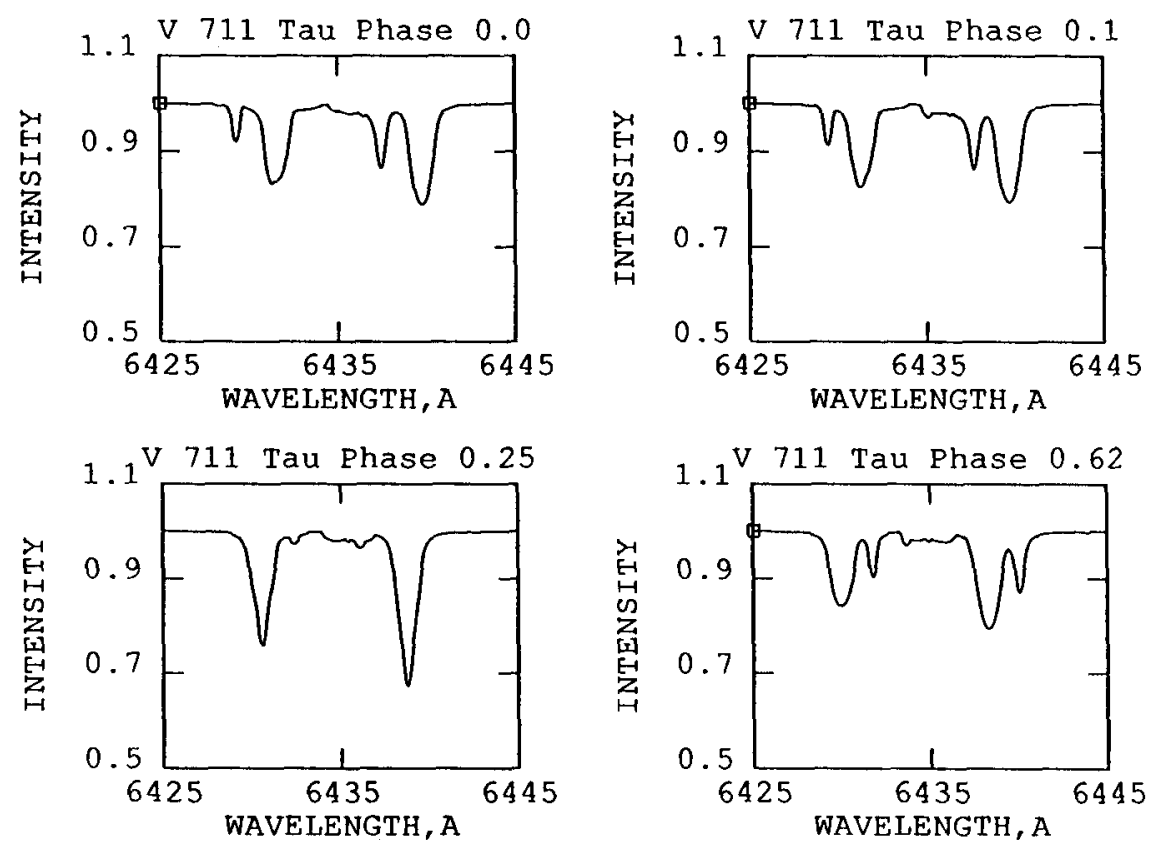

Fig. 1. Examples of the theoretical composite spectra of the HR 1099 system for four orbital phases.

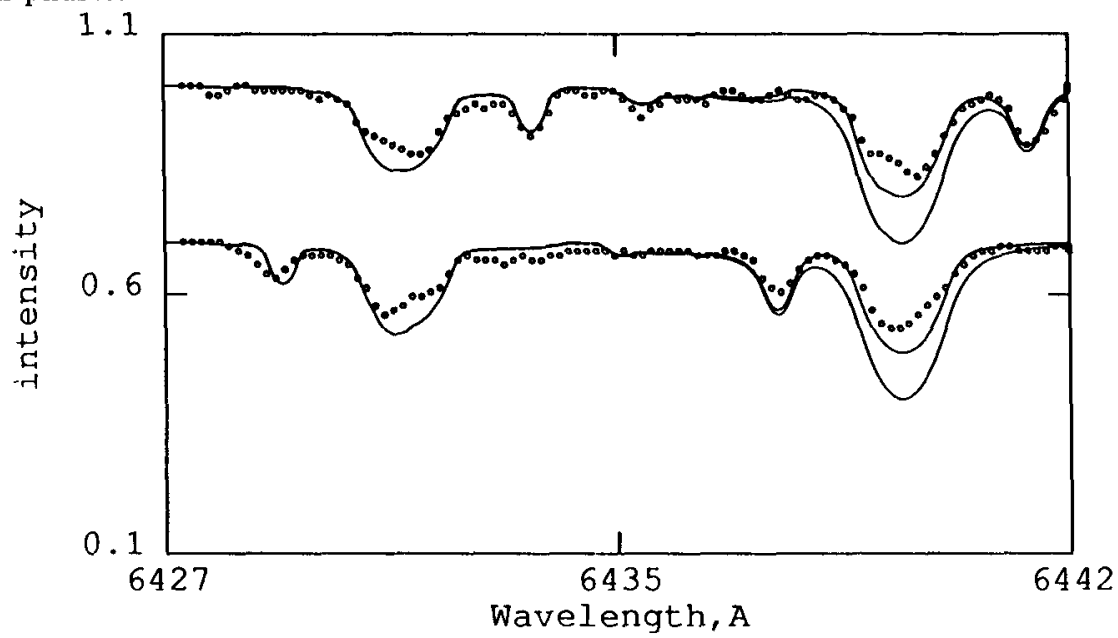

Fig. 2. Comparison between the observed (Fekel, 1983) and calculated spectrum for two phases: 0.565 (top) and 0.919 (bottom). Synthetical spectra are produced for two abundances of $\mathrm{Ca}$ in the atmosphere of the primary: $\log \mathrm{A}(\mathrm{Ca})=5.90$ (upper curve) and $\log \mathrm{A}(\mathrm{Ca})=6.40$ (lower curve). Solar abundances are assumed for other elements in the atmospheres of both components.

the disc centre. This approximation, which should be avoided in the mapping procedures of surface inhomogeneities (see e.g. Piskunov, and Hackman et al., these Proceedings), influences the form of the line profile in the line centre. 
Figure 3 provides the possibility of estimating the influence of microturbulent velocity $\xi_{\mathrm{t}}$ and changes in damping constant $\gamma$ on the $\mathrm{Ca} \mathrm{I}$ and $\mathrm{Fe} I$ lines. The total damping constant in our calculations is assumed to be the sum of radiative, Stark and van der Waals broadening (e.g. Gray, 1976, p. 250), the last used with a scaling factor of 1.5. The behaviour of the line profiles is different because of the different strengths of the lines and stronger saturation of $\mathrm{Ca} I$ line. While damping changes the whole profile, the microturbulent velocity does not influence the wings. For both $\mathrm{Ca} I$ and $\mathrm{Fe} I$ lines, the best fit can be obtained with the classical values of damping and $\xi_{\mathrm{t}}=1.0 \mathrm{~km} / \mathrm{s}$. It can also be concluded that changes in the parameters $\gamma$ and $\xi_{\mathbf{t}}$ can have similar effects to the changes in abundances.
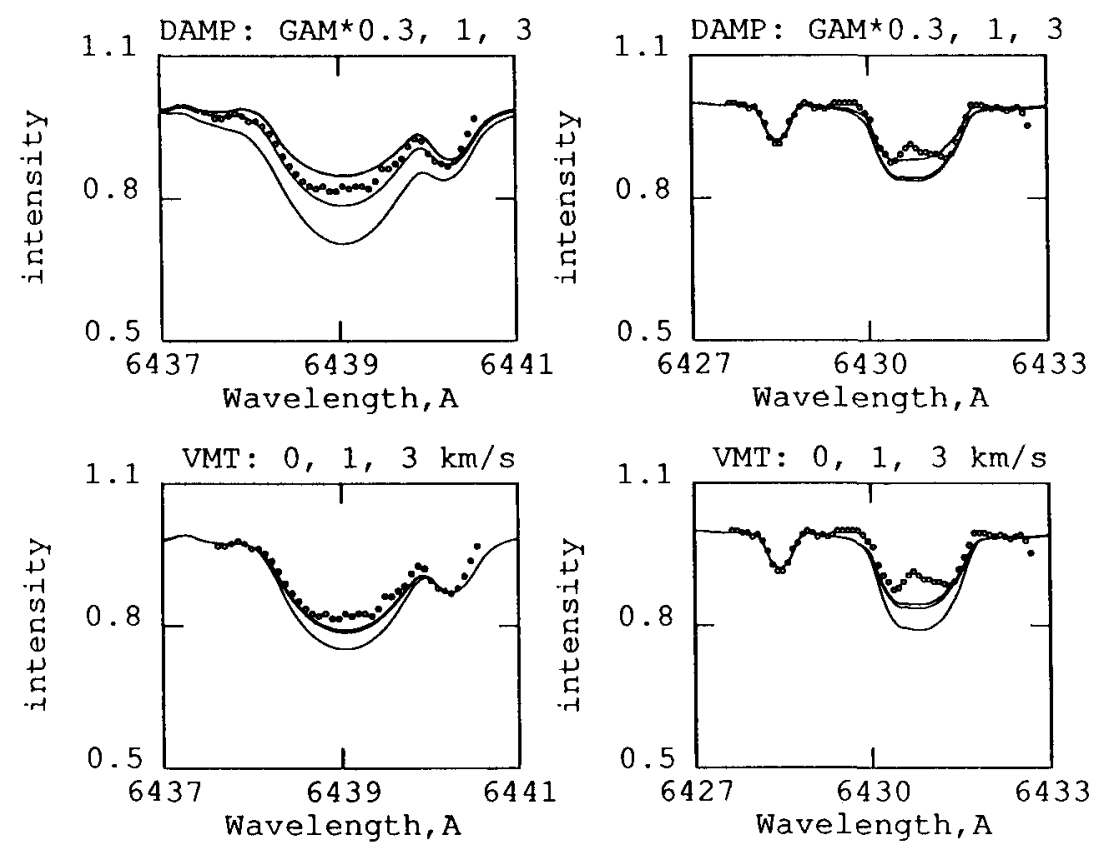

Fig. 3. Comparison between the observed (Vogt, 1983) and synthetic spectrum for Fe I (left) and Ca I (right) lines. Theoretical spectra are calculated with three values of the total damping constant - the "classical" value and this value multiplied by 0.3 and 3 (upper part of the figure). The lower part represents the synthetic spectrum with three values of microturbulent velocity, 0,1 and $3 \mathrm{~km} / \mathrm{s}$.

\section{References}

Bell, R.A., Eriksson, K., Gustafsson, B., Nordlund A.: 1976, Astron. Astrophys. Suppl. 23, 37

Fekel, F.C.: 1983, Astrophys. J. 268, 274

Gray, D.F.: 1976, in The observations and analysis of stellar photospheres, Wiley, New York

Lyubimkov, L.S., Samedov, Z.A.: 1987, Bull. Crimean Astrophys. Obs. 77, 109

Vogt, S.S., Penrod, G.D.: 1983, Publ. A. S. P. 95, 565 